\title{
Spectral effects in a fiber optic Sagnac interferometer due to undesired perturbations
}

\author{
J.L. Arce Diego*, A. Cobo García, C. Jauregui Misas, J. M. López Higuera \\ Photonics Engineering Group -TEISA Department-University of Cantabria \\ Avda. los Castros s/n, E-39005 Santander (Spain)
}

\begin{abstract}
The influence of reciprocal and non-reciprocal perturbations such as magnetic fields, strain, twist and bending on the eigenfrequency bandstructure in a gyroscope, based on a fiber optic Sagnac interferometer have been determined and discussed.
\end{abstract}

Keywords: Fiber-optic interferometer, polarisation, Sagnac interferometer, eigenfrequency spectrum, fiber-optic gyroscope, reciprocal perturbations, non-reciprocal perturbations.

\section{INTRODUCTION}

Sagnac interferometer used as a gyroscope is the basis of an interferometric sensor for measuring mechanical rotations, or electric current by using the Faraday effect. Its reciprocal configuration allows detecting phase delays induced by nonreciprocal effects, while it remains insensitive to reciprocal effects. It is well know that if we consider the absence of movement and perturbations this structure presents an original eigenfrecuency. Several authors have analysed the effect of the Faraday rotation on the frequency bandstructure of a fibre optic gyroscope $e^{1,2,3}$. But to our knowledge, a study of the dependence on such frequencies with all kinds of perturbations has not been made. In this communication, the influence of perturbations with circular and linear birefringence on the gyroscope eigenfrequencies has been studied and analysed. By using a matricial method, which is an extension of Jones method, $4 \times 4$ matrices ${ }^{2}$, the eigenpolarisation modes and their eigenfrequencies are calculated and then the spectral split that depends on the reciprocal and non-reciprocal undergone perturbations, is evaluated. Accumulated effects of magnetic field, linear and circular birefringence, and geometrical rotation have been taken into account.

\section{THEORICAL MODEL}

The loop of a fiber optic Sagnac interferometer is shown in Fig. 1. It consists of an optical transmission fiber closed loop subjected to perturbations of different kinds: linear birefringence, reciprocal circular birefringence, and non-reciprocal circular birefringence due to Sagnac and Faraday effects. In order to analyse such structure, simultaneous waves travelling in opposite directions can be studied by means of $4 \times 4$ matrices. This method is developed as an extension of Jones method. It represents the total electric field by means of a four-component vector composed of the two Jones vectors -reflection and transmission.

*Tel.: +34-942-201545; Fax: +34-942-201873; E-mail: ilarce@teisa.unican.es 


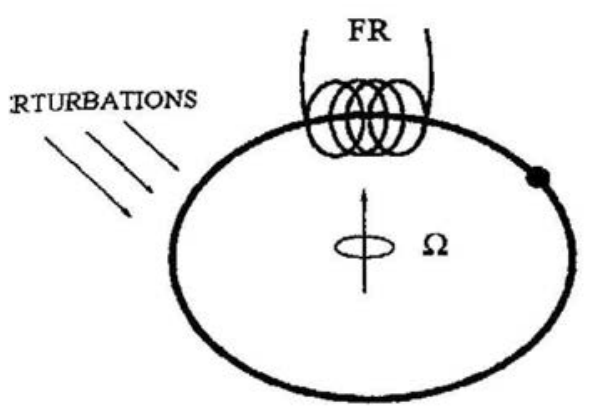

Fig.1. Sketch of the optical fibre loop exposed to a physical rotation( $\Omega$ ), a magnetic freld(FR) and other kinds of perturbations, such as reciprocal circular birefringence and linear birefringence.

nce the full M matrix of the optical fiber loop has been obtained, its eigenmodes are calculated as four vectors which produce themselves according to this equation

$$
\mathbf{M v}=\mathbf{v}
$$

ontrivial solutions to this Equation 1 exist when $\operatorname{det}(\mathbf{M}-\mathbf{I})=0$, being $\mathbf{I}$ the unit matrix. Since $\mathbf{M}$ depends on the frequency, is last expression is the eigenfrequency determining equation. For each eigenfrequency the corresponding eigenpolarization obtained by solving Eq.1, for the eigenvector ${ }^{2}$.

\section{RESULTS AND DISCUSSION}

his section is divided into two parts: In the first place a fiber loop without linear birefringence is analysed, and nally another fiber loop linear birefringence. In both cases the theoretical method that has been previously resented is used.

\section{OPTICAL FIBRE LOOP WITHOUT LINEAR BIREFRINGENCE}

he roundtrip matrix which represents this structure, considering all the different effects, is as follows:

$$
M=e^{j s} \cdot\left[\begin{array}{cc}
\frac{1}{t} \cdot e^{j k L} \cdot m(F+G) \cdot\left(\begin{array}{cc}
1 & 0 \\
0 & 1
\end{array}\right) & j \cdot \frac{r}{t} \cdot e^{-j k L} \cdot m(F+G) \cdot\left(\begin{array}{cc}
-1 & 0 \\
0 & 1
\end{array}\right) \\
j \cdot \frac{r}{t^{*}} \cdot e^{j k L} \cdot m(-F+G) \cdot\left(\begin{array}{cc}
1 & 0 \\
0 & -1
\end{array}\right) & \frac{1}{t^{*}} \cdot e^{-j k L} \cdot m(-F+G) \cdot\left(\begin{array}{ll}
1 & 0 \\
0 & 1
\end{array}\right)
\end{array}\right]
$$

here $\mathrm{L}$ is the roundtrip length of the optical fibre loop, $\mathrm{r}$ and $\mathrm{t}$ are the reflection and transmission coefficients of the ackscatterer, $m$ is the Faraday matrix, where $F$ is the Faraday effect and $G$ the reciprocal circular birefringence. $S$ represents le angular phase induced by the rotation, whose expression is:

$$
S=2 \cdot \frac{\omega}{c^{2}} \cdot \Omega \cdot A_{e f f}
$$

There $A_{\text {eff }}$ is the effective area enclosed by the loop, $\Omega$ is the angular velocity vector of the structure. The influence of any $f$ these perturbations over the fibre loop can be analysed in terms of the eigenfrequencies of the roundtrip matrix of the tructure. Their values correspond to opposite circular polarisation for waves running in opposite directions, and which orresponding eigenfrequencies can be obtained by means of the equations presented in the Tablel. 


\begin{tabular}{|c|c|}
\hline cw rcp & $(k L-\tau)+G+\operatorname{Cos}^{-1}[|t| \operatorname{Cos}(S-F)]=0$ \\
cw lcp & $(k L-\tau)-G+\operatorname{Cos}^{-1}[|t| \operatorname{Cos}(S+F)]=0$ \\
$\operatorname{ccw~rcp}$ & $(k L-\tau)+G+\operatorname{Cos}^{-1}[t \mid \operatorname{COS}(-S+F)]=0$ \\
$\operatorname{ccw~lcp}$ & $(k L-\tau)-G+\operatorname{Cos}^{-1}[|t| \operatorname{Cos}(-S-F)]=0$ \\
\hline
\end{tabular}

Table 1. Corresponding eigenfrequencies to the four optical waves according to the sense of propagation (cw or ccw) and circular polarisation (rcp or lcp)

where cw and ccw refers to clockwise and counter clockwise travelling waves respectively, S is the Sagnac phase angle, F the Faraday rotation, $k$ the wave number and $L$ is the roundtrip length, with reciprocal birefringence $G$ and $\tau=\arg (t)$.

The effect of perturbations on the frequency response is now considered. The existence of circular birefringence, reciprocal and non reciprocal, in an optic fibre loop produces the oscillation of four waves, two of them in clockwise sense (cw) and the other two in counter clockwise sense (ccw), with right hand (rcp) or left hand (lcp) circular polarisation ${ }^{3}$.

In an isotropic cavity, in the absence of any kind of perturbation, the resonance frequency is given by Eq. 4 , where $\mathrm{m}$ is an integer, $\mathrm{c}$ the velocity of light in vacuum, and $\mathrm{L}$ the roundtrip length.

$$
v_{0}=\frac{\omega}{2 \pi}=\frac{k L}{2 \pi} \frac{c}{L}=m \frac{c}{L}
$$

The effect produced by the different perturbations over the four components of the signal which are copropagating into the cavity is exposed in Table 2.

\begin{tabular}{lcccc}
\hline & $\mathrm{cw}-\mathrm{rcp}$ & $\mathrm{cw}-\mathrm{lcp}$ & $\mathrm{ccw}-\mathrm{rcp}$ & $\mathrm{ccw}-\mathrm{lcp}$ \\
\hline Sagnac: $\mathrm{S}$ & $+\mathrm{S}$ & $+\mathrm{S}$ & $-\mathrm{S}$ & $-\mathrm{S}$ \\
Non-reciprocal: $\mathrm{F}$ & $-\mathrm{F}$ & $+\mathrm{F}$ & $+\mathrm{F}$ & $-\mathrm{F}$ \\
Reciprocal: $\mathrm{G}$ & $+\mathrm{G}$ & $-\mathrm{G}$ & $+\mathrm{G}$ & $-\mathrm{G}$ \\
\hline
\end{tabular}

Table 2. Sign of the phase change produced in every kind of perturbation according to the sense of propagation (cw or ccw) and circular polarisation (rcp or lcp)

The split of an original resonance frequency into four frequencies, giving the spectrum that Fig. 2 shows can be explained, in agreement with Table 1, as follows:

a) The exposition to an axial magnetic field introduces a phase difference between the oscillation of two circular modes of light. Since the Faraday effect is non-reciprocal, the waves propagating in opposite directions undertake a phase difference with opposite signs between their circular polarisation modes. Such phase difference is given by:

$$
\Delta v_{F}=\frac{c}{L} \frac{F}{2 \pi}
$$


b) If the effect of reciprocal circular birefringence along the optical path is introduced, each of the four waves experiences a slight optical path difference, that produces an additional frequency separation that has positive sign for rcp waves and negative sign for lcp waves. Such separation can be expressed as

$$
\Delta v_{c}=\frac{c}{L} \frac{G}{2 \pi}
$$

c) If the structure is subjected to physical rotation, the frequency separation between the rcp waves that travel clockwise and counterclockwise decreases $\Delta v_{s}$, while the separation between the lcp waves increases $\Delta v_{s}$, where $\Delta v_{s}$ is:

$$
\Delta v_{s}=\frac{c}{L} \frac{S}{2 \pi}
$$

a)

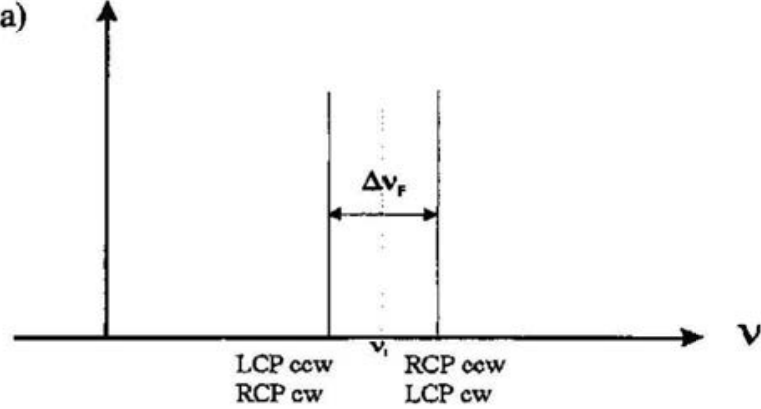

c)

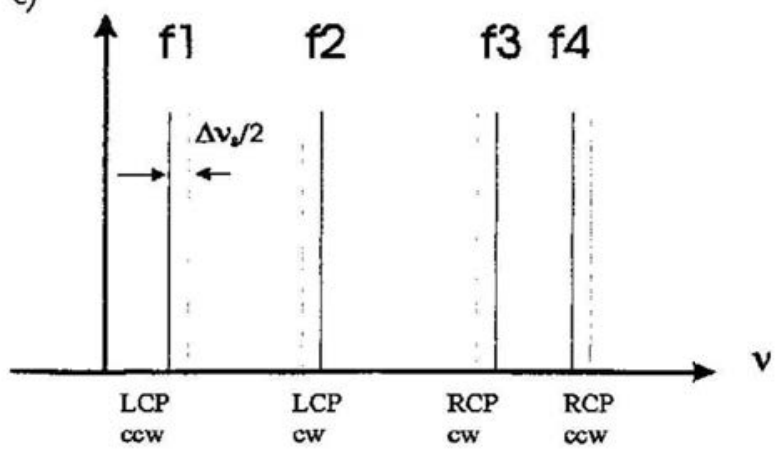

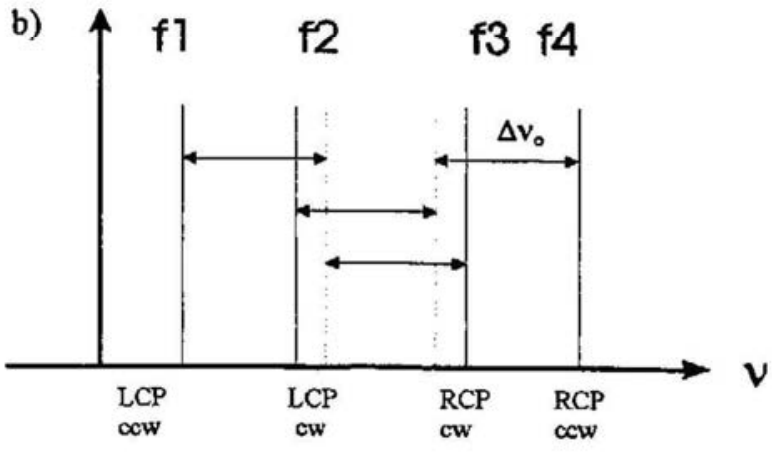

Fig.2. Eigenfrequency spectrum evolution in a Sagnac interferometer. Accumulated effects of circular birefringence, a) magnetic field, b)

The electric current amplitude I, in the solenoide of Fig. 1 that produce the spectral displacements depicted in Fig. 2 have been obtained by means of signal processing. In Fig. 3 the relation between electric current in kA and the frequency displacement, for constant values of Sagnac phase ( $\mathrm{S}=1 \mathrm{rad})$ and phase difference due to circular birefringence $(\mathrm{G}=5 \mathrm{rad})$ and linear birefringence $(\mathrm{b}=0.1 \mathrm{rad})$ is displayed. The phase correspondent to the resonant frequency in the absence of perturbations has been considered to be $2.5 \mathrm{rad}$, and the operation wavelength was $1553 \mathrm{~nm}$. A frequency displacement of $2.268 / \mathrm{L} \mathrm{Hz} / \mathrm{A}$ is appreciated, $\mathrm{L}$ being the fiber interferometer length in meters. Besides, the frequency displacement behaviour in the presence of electric current matches that expected following Fig. 3. 


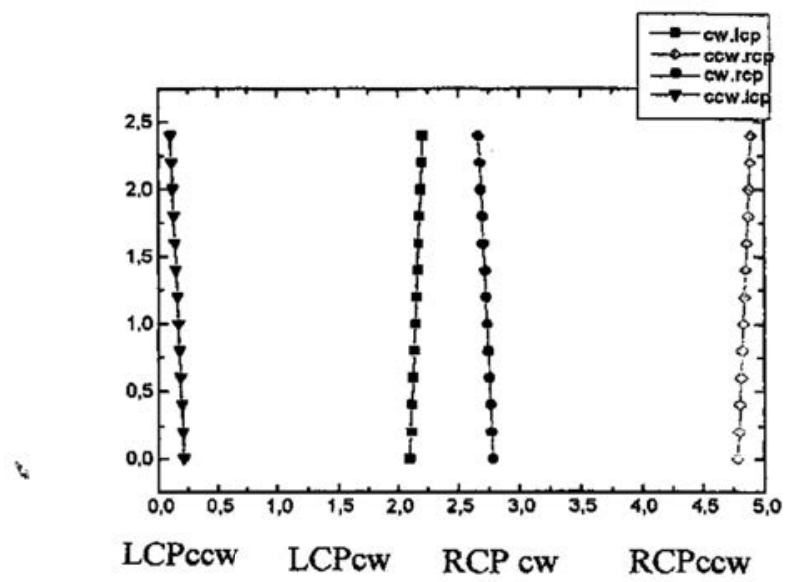

Fig.3. Distribution of a Sagnac interferometer frequency lines as a function of the electric current on a 50-coils solenoid, considering the phase difference between eigenmodes that is due to a Sagnac effect of $\mathrm{S}=1 \mathrm{rad}$, a circular birefringence value of $\mathrm{G}=5 \mathrm{rad}$,

\subsection{OPTICAL FIBRE LOOP WITH LINEAR BIREFRINGENCE}

If the fibre loop has an accumulated lineal birrefringence $b$ which is different to zero, then, the eigenmodes of polarisation are eliptics, the corresponding eigenfrequencies can be evaluated by using the Table.3, and the $M$ matrix in the case of a freereflections fibre loop can be separated into two Jones matrices and their expressions for $\mathrm{cw}$ and ccw directions are:

$$
\begin{aligned}
& M_{T}^{c \omega}=\frac{1}{t} \cdot e^{i k L} \cdot e^{i s} \cdot\left[\begin{array}{cc}
e^{i b} & 0 \\
0 & e^{-i b}
\end{array}\right] \cdot\left[\begin{array}{cc}
\cos (F+G) & -\sin (F+G) \\
\sin (F+G) & \cos (F+G)
\end{array}\right] \\
& M_{T}^{c c \omega}=\frac{1}{t^{*}} \cdot e^{i k L} \cdot e^{i s} \cdot\left[\begin{array}{cc}
e^{-i b} & 0 \\
0 & e^{i b}
\end{array}\right] \cdot\left[\begin{array}{cc}
\cos (F-G) & \sin (F-G) \\
-\sin (F-G) & \cos (F-G)
\end{array}\right]
\end{aligned}
$$

$$
\begin{array}{lll}
\text { cw rep } & \Rightarrow & k L+S-\operatorname{Cos}^{-1}[\operatorname{Cos}(F-G) \operatorname{Cos} b]=0 \\
\text { cw lep } & \Rightarrow & k L+S+\operatorname{Cos}^{-1}[\operatorname{Cos}(F-G) \operatorname{Cos} b]=0 \\
\text { ccw rep } & \Rightarrow & k L-S+\operatorname{Cos}^{-1}[\operatorname{Cos}(F+G) \operatorname{Cos} b]=0 \\
\text { ccw lep } & \Rightarrow & k L-S-\operatorname{Cos}^{-1}[\operatorname{Cos}(F+G) \operatorname{Cos} b]=0
\end{array}
$$

Table 3. Corresponding eigenfrequencies to the four optical waves according to the sense of propagation (cw or ccw) and elliptical polarisation (rep or lep)

An additional spectrum shift is introduced as a consequence of linear birefringence in optical fibres. This frequency shift between eigenmodes travelling in opposite direction and with the same kind of polarisation becomes in: 